And the remaining four workshop could reach at 1.7044, $2.0238,1.3152$ and 1.2136 times respectively after adjusting each component according to the parameter. The adjusted data were put into the occupational input-output table and the prediction model was built.

Conclusion If the estimated annual output of a given year is known, after being converted to the total output value, the most suitable occupational health input and the largest occupational health output can be calculated.

\section{THE ASSOCIATION BETWEEN WORK-FAMILY SPILLOVER AND QUALITY OF SLEEP: A PROSPECTIVE STUDY OF JAPANESE WORKERS}

\begin{abstract}
${ }^{1}$ Yui Hidaka*, ${ }^{2}$ Akihito Shimazu, ${ }^{3}$ Kotaro Imamura, ${ }^{4}$ Noboru Iwata, ${ }^{5}$ Kyoko Shimada, ${ }^{6}$ Masaya Takahashi, ${ }^{7}$ Masahito Tokita, ${ }^{8}$ Izumi Watai, ${ }^{3}$ Kazuhiro Watanabe, ${ }^{3}$ Norito Kawakami. ${ }^{7}$ Department of Psychiatric Nursing, Graduate School of Medicine, The University of Tokyo, Tokyo, Japan; ${ }^{2}$ Center for Human Social Science, Kitasato University College of Liberal Arts and Science, Sagamihara, Japan; ${ }^{3}$ Department of Mental Health, Graduate School of Medicine, The University of Tokyo, Tokyo, Japan; ${ }^{4}$ Department of Psychology, Hiroshima International University, Hiroshima, Japan; ${ }^{5}$ Department of Social Psychology, Faculty of Sociology, Toyo University, Tokyo, Japan; ${ }^{6}$ National Institute of Occupational Safety and Health, Kawasaki, Japan; ${ }^{7}$ Department of Psychiatric and Mental Health Nursing, Graduate School of Nursing, Chiba University, Chiba, Japan; ${ }^{8}$ Department of Nursing, Graduate School of Medicine, Nagoya University, Nagoya, Japan
\end{abstract}

\subsection{6/oemed-2018-ICOHabstracts.485}

Introduction Quality of Sleep has been linked to poor health. While work-family spillover is associated with health problems and impaired work performance of workers, previous findings were inconsistent on, the association between work-family spillover and sleep quality. No study was conducted in nonwestern countries. The objective of this study was to investigate the prospective association between work-family spillover and sleep quality among Japanese workers.

Methods The database used in the study was from a 2 year prospective cohort study. In the baseline survey (T1) in 2011, questionnaires were sent to 1356 workers living in two wards of Tokyo, and 753 (56\%) responded, and 489 completed all scales and items used the study. A follow-up survey was conducted in 2013, with 224 (46\%) respondents out of the 489 completers at T1; 176 completed all scales. The questionnaire (both T1 and T2) included self-reported instruments of workfamily spillover (the Survey Work-home Interaction-NijmeGen, SWING), sleep quality (Pittsburgh Sleep Quality Index, PSQI), and job stressors (Brief Job Stress Questionnaire, BJSQ), and demographic variables (age, sex, education, marital status, and work styles). Multiple linear regression analysis was employed of PSQI scores on SWING scale scores, adjusting for demographic variables and PSQI score at T1. (SPSS version 22)

Results Among the 176 completers, 36\% were men; the average age was 39 years old. Work to family negative spillover (beta $=0.20)$ significantly and positively correlated with sleep quality, after adjusting for the demographic variables $(p=0.020)$. No significant association was observed between the other type of spillover (i.e. work to family positive, family to work positive or negative) and sleep quality. ( $p>0.05)$.

Conclusion This prospective study confirmed the association between work-family negative spillover and sleep quality in a sample of Japanese workers. Work to family negative spillover could be considered a target condition to improve sleep quality of workers.

\section{HEALTH LITERACY IN JAPANESE WORKPLACE (2ND REPORT): IMPACT OF WORKPLACE HEALTH PROMOTION, LESSONS LEARNED FROM THE PRACTICE}

${ }^{1}$ Hiroshi Fukuda*, ${ }^{1}$ Fumiko Kitajima, ${ }^{2}$ Yuka Sakamoto, ${ }^{2}$ Kayo Suwa, ${ }^{2}$ Akiko Fujiwara, ${ }^{2}$ Rie Morita. ${ }^{1}$ Department of General Medicine, School of Medicine, Juntendo University, Tokyo, Japan; ${ }^{2}$ Nikken Sekkei LTD, Tokyo, Japan

10.1136/oemed-2018-ICOHabstracts.486

Introduction Integration of Occupational Health and Safety (OHS) and Workplace Health Promotion(WHP) aiming at employees' health and well-being attracts attention in WHO's 'Healthy Workplace Framework' and NIOSH's 'Total Worker Health'. Also in Japan, WHP is actively conducted in many companies in trends, however there are few studies on the effectiveness of WHP in Japan. We examined the impact on its lifestyle and health literacy and success factors from the practical experience of WHP in a company.

Methods We reported OHS and WHP activities in an architectural consultant company for 15 years, and its impact in the pre-post comparison design to study its success factors.

Results OHS and WHP activities at the company began in 2001. It inducted occupational physician (OP)/OHS committee (2001); employment of occupational health nurse (OHN)/ developed guidelines (2002); e-mail newsletters (2003); first health survey (2005), full-time employment of OHN(2006), healthy lunch box project (2007), OHSMS (2009); health policy (2011); health lecture/portal web-site (2012); mental health training/company sports festival (2013); health lecture by labour union(2014); gymnastics with the president/3rd health survey (2015), delivery of health lecture to each department (2016), etc. In the pre-post comparison of lifestyles using health survey, employees thinking nutrition balance $(40 \rightarrow 79 \%)$, having exercise habits $(31 \rightarrow 36 \%)$ and sufficient sleep $(12 \rightarrow 18 \%)$ increased, improving the employee's health literacy for four consecutive years from 2014 to 2017.

Conclusion A good impact on lifestyle and health literacy was obtained from 15 years of WHP. It was considered that success factors of WHP practice involving human resources including management/labour union are:

1. discovery of key persons highly interested in health,

2. existence of $\mathrm{OHN}$,

3. corporate culture with good communication, and

4. clear management's commitment and health policy.

WHP will be increasingly important in Japan as ageing population and health disparities progress. Its important outcome is organisational and employee health literacy, so we want to continue the research to contribute to its improvement.

\section{FUTURE CHALLENGES FOR THE OCCUPATIONAL PHYSICIAN}

${ }^{1} \mathrm{P}$ Swennen*, 'S Acke, ${ }^{1,2} \mathrm{M}$ Verbrugghe, ${ }^{1} \mathrm{M}-\mathrm{N}$ Schmickler, ${ }^{3} \mathrm{E}$ Schleich. ${ }^{1} M e n s u r a$ Occupational Health Services, Belgium; ${ }^{2}$ Department of Public Health, University of Ghent, Belgium; ${ }^{3}$ Société Scientifique de Santé au Travail, Belgium

\subsection{6/oemed-2018-ICOHabstracts.487}

Introduction An economic and demographic transition with an ageing workforce, evolving work organisation and new emerging risks is combined with a shortage of Occupational Physicians (OPs). This study aims to propose recommendations for 
future occupational health surveillance (OHS) and the role of OPs.

Methods First, the PubMed, EU-OSHA and International Labour Organisation databases were screened for literature from 2006 to August 31, 2016, concerning OHS, return-towork policies and risk management systems in Europe, Japan, Canada and other OECD countries. Second, a standardised mail survey was delivered to all members of the Belgian Professional Association of OPs.

Results

- The literature review showed that European directives legally inspired countries to adapt their legislations and develop strategic OHS plans. Nevertheless, the comparison of OHS in different countries shows a varied landscape.

- The response rate of the survey was $54 \%(n=262)$.

The results prompt a paradigm shift:

a. Within 5 years, OPs must focus more on return-to-work (96.3\%; 95\% CI: $93.2 \%$ to $98.1 \%$ ) and risk analyses $(84.9 \%$; 95\% CI: $79.9 \%$ to $88.8 \%$ ) while training occupational nurses (ONs) to execute routine occupational health examinations (46\%; 95\% CI: $39.8 \%$ to $52.4 \%$ ). Similarly, a more standardised and multidisciplinary approach to risk assessment should be developed (74.7\%; 95\% CI: $68.8 \%$ to $79.8 \%)$.

b. By 2030, OPs would be more specialised in toxicology, disability management, ionising radiation, etc. (42.2\%; 95\% CI: $36.2 \%$ to $48.5 \%$ ), and ONs would be trained on risk assessments (74.8\%; 95\% CI: $68.9 \%$ to $79.9 \%)$ and health promotion (98\%; 95\% CI: $95.3 \%$ to $99.1 \%)$. OPs disagree that general practitioners (GPs) could solely execute occupational health examinations $(81.7 \%$; $95 \% \mathrm{CI}$ : $76.4 \%$ to $86.1 \%)$.

Discussion The role of OPs must be part of a trend toward evidence-based OHS (i.e., studying the effectiveness and efficiency of 'fitness for the job assessments'), disability management and engagement with a wide network of stakeholders (such as ONs, GPs and health insurance organisations).

\section{RESPONSES FROM 'HIGH-STRESS' WORKERS OF THE STRESS CHECK PROGRAM IN JAPAN - A CASE STUDY}

H Tahara* , H Hiro. University of Occupational and Environmental Health, Japan, Kitakyushu, Japan

\subsection{6/oemed-2018-ICOHabstracts.488}

Introduction The Stress Check Program in Japan (SC), based on the Industrial Safety and Health Law, began on Dec 1, 2015. It requires an employer to

1. provide a survey of psychosocial stress for workers,

2. report to each individual worker his/her results,

3. arrange for an interview by a physician when requested to do so by a worker with high stress,

4. consider the opinions of the physician and improve working conditions for the worker, and

5. not take any action against the worker because he/she needs changes of his/her working conditions (Kawakami and Tsutsumi, 2016).

Ministry of Health, Labour and Welfare, Japan (MHLW) recommends an employer to assign an occupational health physician (OHP) to both the designated staff of the survey (DSS) and the interviewer (IVW).

Methods In June 2017, the Brief Job Stress Questionnaire (Shimomitsu, et al., 2000) was used as the survey at several offices of a company in Japan, where one of the presenters is assigned to OHP, DSS, and IVW. The criteria for 'high-stress' was based on an example appeared in the manual by MHLW. A presenter (as DSS) sent e-mail to fifty 'high-stress' workers, offering the interview by him (as IVW) and promoting permanent health consultation services by him (as OHP) or by outsourcing psychologists. Then he described the responses from the workers for a month.

Results Three workers requested the interview. Other ten workers reported his/her state of health by e-mail, phone or permanent health consultation. Another worker was accidentally arranged health consultation by the boss, not reported the result of the survey, due to sickness absence.

Conclusion Though SC as single program has weak scientific evidence for reducing workers' mental health problems; it may become more effective as a part of continuous occupational health promotion.

\section{PROCESS OF ACCREDITATION OF HEALTH SERVICE IN AUTOMOTIVE COMPANY OF BRAZIL}

${ }^{1}$ Paulo Zetola, ${ }^{2}$ Ana Claudia Soares, ${ }^{2}$ Livia Schwab, ${ }^{2}$ Jose Ari Bastos, ${ }^{2}$ Wilson Demarchi. ${ }^{1}$ Universidade Federal Do Paraná, Curitiba, Brazil; ${ }^{2}$ Medical Service Renault Automotive, Curitiba, Brazil

\subsection{6/oemed-2018-ICOHabstracts.489}

Introduction Private and public companies that have employees governed by the Consolidation of Labour Laws must have Specialised Services in Safety and Occupational Medicine, with the purpose of promoting health and protecting the integrity of the worker. These health professionals are responsible for urgent and emergency care in the work environment where we know the need for technical training of the professionals. The problem is that many find themselves without adequate updating in scientific training, updating in their internal processes, risk management that guarantees a safe assistance to the worker. These are the challenges of this research, identifying which are the main difficulties encountered by health managers of the company to implement an accreditation process in their medical services.

Method Cross-organisational study developed through the data and indicators obtained with the implementation of an Accreditation and Certification process of the National Accreditation Organisation (ONA) in Brazil, from 2014 to 2017.

Results The implementation of the Accreditation process in the company's Medical Service allowed us to identify many process failures in the service routines. Pharmacy, Ambulatory Infection, and Medical Records Committees were establish based on the requirements of the Accreditation and Certification Standards. All employees attended received an adhesive label with his name to be stitch to the clothing. Urgent care protocols were develop, as well as the Manchester Protocol for the prioritisation of care. A worksheet was create to identify all the Adverse Events found and a follow-up list of the pending action plans.

Discussion The Certification process is a significant differential for companies that are ready to implement it as it assures the patient's life and reduces the chances of medical failures or processes that generate damages to the patient. This company 